\title{
Vesicoperitoneal fistula: A rare late complication of laparoscopic removal of intrauterine device
}

\author{
Oktay Ucer ${ }^{1 *}$, Mehmet Bilgehan Yuksel ${ }^{1}$, Gokhan Temeltas ${ }^{1}$, Talha Muezzinoglu ${ }^{1}$
}

\author{
Abstract \\ We present a case of vesicoperitoneal fistula occurring 12 years after laparoscopic removal of the intrauterine \\ device.
}

A 30-year-old primiparous woman presented with acute abdominal pain. On examination, there were sensitivity and painful of all the abdomen quadrants on gentle palpation. The diagnostic workup included transabdominal ultrasonography and contrast computed tomography that revealed leakage of contrast into the peritoneal cavity from the bladder. At laparotomy, the fistula tract was excised.

Vesicoperitoneal fistula is a rare major complication of laparoscopic surgeries and occurs in early postoperative period. We observed the vesicoperitoneal fistula as a late complication of laparoscopic approach in the present case. This may be due to the thinning of a urachal diverticulum wall in the bladder during the laparoscopic procedure.

Keywords: Complication, Laparoscopy, Vesicoperitoneal fistula.

\section{Introduction}

A vesicoperitoneal fistula is an epithelialized communication between the bladder and peritoneal cavity. It is an extremely rare condition and occurs as an early complication of obstetric or gynaecological interventions (1). We present a case of vesicoperitoneal fistula as a late complication of laparoscopy that occurred 12 years after laparoscopic removal of the intrauterine device

Case

A 30-year-old primiparous woman was presented to the department of emergency service at our hospital with acute abdominal pain. The surgery history of the patient was only the presence of a laparoscopic removal of the intrauterine device 12 years ago. She had no any history of recurrent paint, recurrent urinary tract infection, and gastrointestinal discomfort. Abdominal ultrasonography and computed tomography revealed intra-abdominal free fluid collection and no evidence of intra-abdominal organ injury.
She was referred to our clinic with the suspicion of bladder perforation. On examination, there were sensitivity and painful of all the abdomen quadrants on gentle palpation. Computed tomographic cystography demonstrated a leakage of contrast into the peritoneal cavity from posterosuperior wall of the bladder (Figure 1A).

A foley catheter was inserted into the bladder of the patient and she was hospitalized in our clinic. At laparotomy, the vesicoperitoneal fistula tract was excised, and peritoneum and bladder were sutured separately. The median umbilical ligament was intact. The beginning of the fistula tract was just below the location that the entry into the bladder of this ligament. The postoperative period of the patient was uneventful. The patient was examined 2 weeks after the procedure. Postoperative cystography revealed none leakage of contrast into the peritoneal area (Figure 1B). So, the foley catheter was removed. There has been no sign of fistula and the patient remains asymptomatic for 3 months. The patient provided written consent to use the information for the case report.

Received: 11-12-2015, Accepted 29-12-2016, Available Online 15-03-2016

1 Celal Bayar University, Faculty of Medicine, Department of Urology, Manisa, Turkey

*Corresponding Author: Oktay Ucer E-mail: uceroktay@yahoo.com 

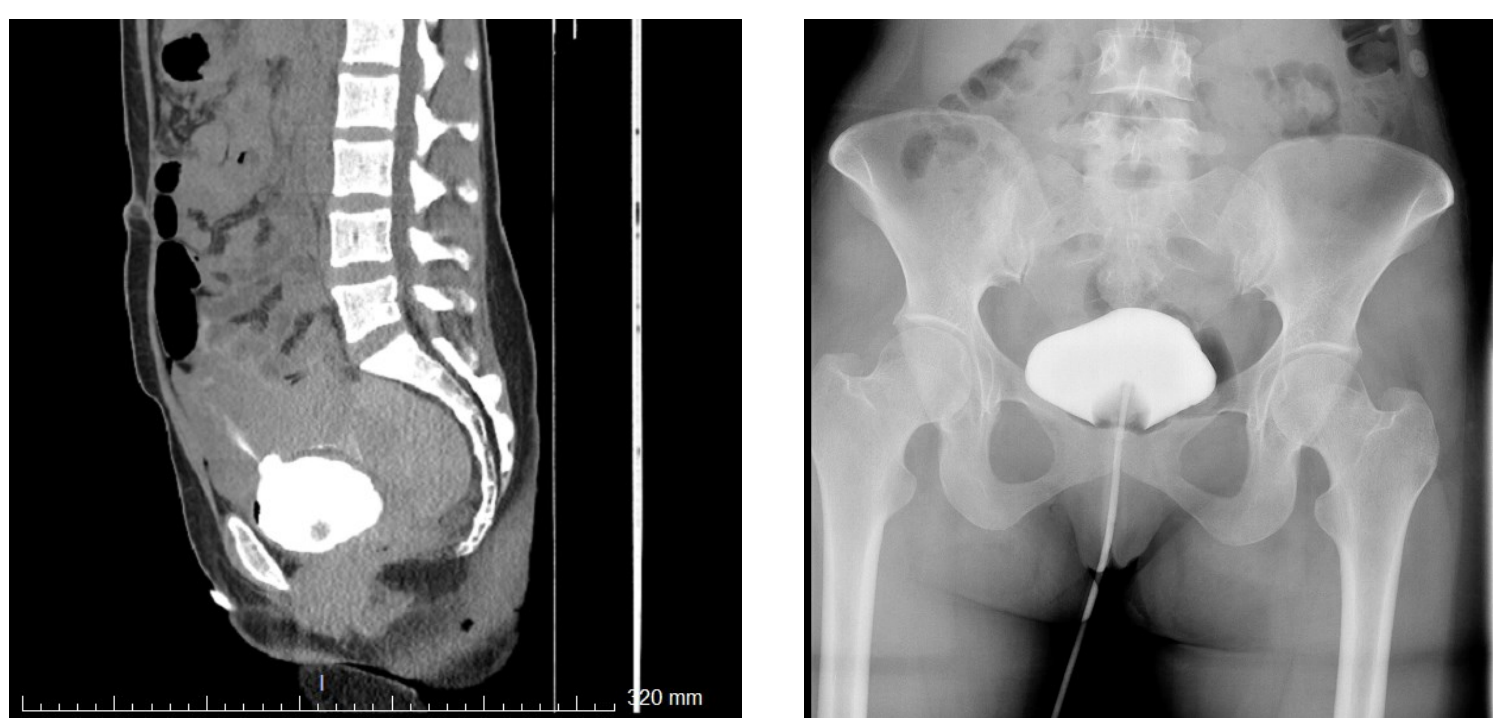

Figure 1. A: The leakage of contrast into the peritoneal area from the bladder in the preoperative computed tomographic cystography. B: The view of the bladder in the cystography two weeks after the operation.

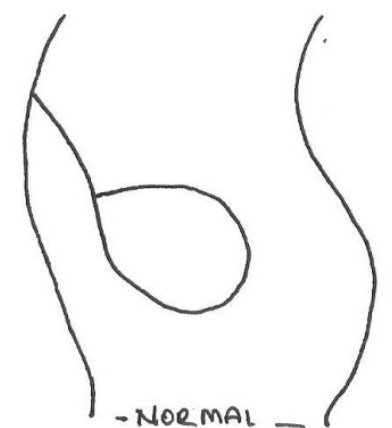

(A)

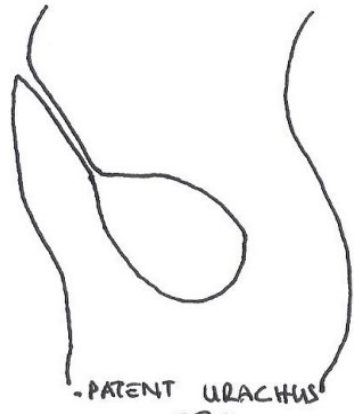

(B)

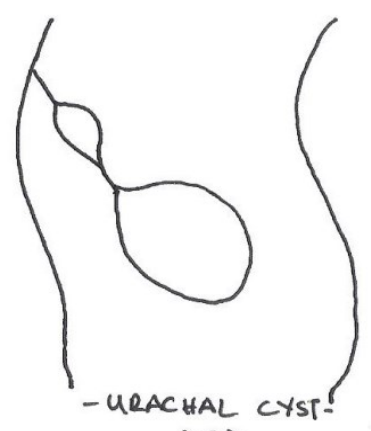

(C)

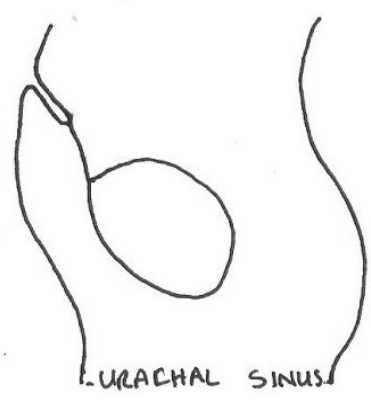

(D)

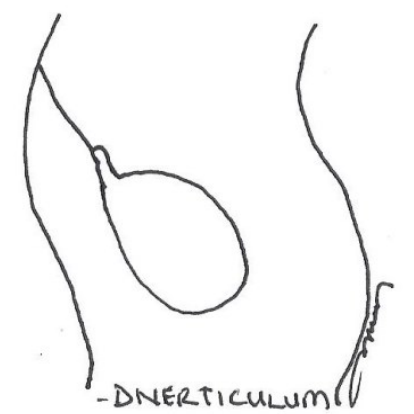

(₹)

Figure 2. Urachal abnormalities (A: Normal, B: Patent urachus, C: Urachal cyst, D: Urachal sinus, E: Urachal diverticulum) 


\section{Discussion}

Laparoscopy is now widely recognized as an indispensable tool in gynaecologic surgery. The incidence of vesicovaginal fistula after laparoscopic hysterectomy is approximately 1 in $455(2,3)$. Whereas, rates of vesicoperitoneal fistula after laparoscopic surgery remain unclear. Donnez et al (3) observed only one vesicoperitoneal fistula in their series of 3190 laparoscopic hysterectomies $(0,06 \%)$. The vesicoperitoneal fistula was diagnosed 6 days after surgery. The patient presented with acute pelvic pain and ultrasound revealed the presence of liquid in the peritoneal area. The diagnosis of the fistula was made by computed tomography scan. A Foley catheter was left in place for 14 days. Only two patients with vesicoperitoneal fistulas reported in the literature to date. The traumatic vesicoperitoneal fistula presented within days with acute onset abdominal pain following emergency caesarean section. Although iatrogenic bladder injury may have occurred in this case, it remains a matter of speculation (4). The other case presented with chronical abdominal pain one year after an emergency caesarean section for fetal bradycardia (5).

Vesicoperitoneal fistula is a rare major complication of laparoscopic surgeries and occurs in early postoperative period. To our best knowledge, this is first case of vesicoperitoneal fistula that occurred a long time (12 years) after a laparoscopic surgery in literature to date. In the medical history of the patient, there were no surgeries or diseases except the removal of intrauterine device. The beginning of the fistula was at posterosuperior of the bladder and just below the median umbilical ligament. We think that the vesicoperitoneal fistula might be due to the thinning of a urachal diverticulum wall (Figure 2) in the bladder during the umbilical port insertion in the laparoscopic procedure.

\section{Conclusion}

Vesicoperitoneal fistula may be rarely experienced as a late postoperative complication of laparoscopic surgery. We should consider this issue in patients with acute abdominal pain and a history of laparoscopic surgery

\section{Acknowledgements: None}

Funding: None Declared

Conflict of Interest: The authors declare no potential conflicts of interest with respect to the research, authorship, and/or publication of this article.

\section{References}

1. Kelly J. Repair of obstetric fistulae: review from an overseas perspective. The Obstetrician and Gynaecologist 2002; 4: 205-11.

2. Harkki-Siren $\mathbf{P}$, Sjoberg $\mathbf{J}$ and Titinen A. Urinary tract injuries after hysterectomy. Obstet Gynecol 1998; 92: 113

3. Donnez O, Jadoul P, Squifflet J, et al. A series of 3190 laparoscopic hysterectomies for benign disease from 1990 to 2006: evaluation of complications compared with vaginal and abdominal procedures. BJOG 2009; 116(4): 492-500.

4. Ismail S. A vesico-peritoneal fistula presenting with pain. J Obstet Gynaecol 2007; 27(2): 194

5. Ooi WL, Sherwood BT, Stanley J, et al. Vesico-peritoneal fistula: a rare cause of chronic abdominal pain. Journal of Clinical Urology 2014; 7(2): 121-2. 